\title{
DESIGN CONSIDERATIONS OF A MW-SCALE, HIGH-EFFICIENCY, INDUSTRIAL-USE, ULTRAVIOLET FEL AMPLIFIER
}

\author{
C. Pagani, INFN Milano - LASA, Via Cervi, 201, 20090 Segrate (MI), Italy \\ E.L. Saldin, E.A. Schneidmiller, DESY, 22607 Hamburg, Germany \\ M.V. Yurkov, Joint Institute for Nuclear Research, Dubna, 141980 Moscow Region, Russia
}

\begin{abstract}
Theoretical and experimental work in free electron laser (FEL) physics, and the physics of particle accelerators over the last ten years has pointed to the possibility of the generation of MW-level optical beams with laser-like characteristics in the ultraviolet (UV) spectral range. The concept is based on generation of the radiation in the master oscillator - power FEL amplifier (MOPA) configuration. The FEL amplifier concept eliminates the need for an optical cavity. As a result, there are no thermal loading limitations to increase the average output power of this device up to the MW-level. The problem of a tunable master oscillator can be solved with available conventional quantum lasers. The use of a superconducting energy-recovery linac could produce a major, cost-effective facility with wall plug power to output optical power efficiency of about 20 per cent that spans wavelengths from the visible to the deep ultraviolet regime.
\end{abstract}

\section{INTRODUCTION}

Significant efforts of scientists and engineers working in the field of conventional quantum lasers are directed towards the construction of powerful ultraviolet (UV) lasers for industrial applications such as material processing, lithography, isotope separation, and photo-induced chemistry. Nevertheless, this problem is still unsolved: progress in this field is rather moderate and we cannot expect a significant breakthrough in the near future.

Recent investigations have shown that this problem can be solved by free electron lasers (FELs), which can be divided into two classes: amplifiers and oscillators. FEL amplifiers amplify the input electromagnetic wave from the external master oscillator. The FEL oscillator can be considered as an FEL amplifier with feedback, which is carried out by means of an optical resonator. Recently a $\mathrm{kW}$-scale UV FEL oscillator project has been initiated at Jefferson Laboratory [1,2].

The main problem of constructing a high average power UV FEL oscillator is the heating effects in the cavity mirrors. The results of optical resonator modeling have demonstrated that the oscillator scheme for UV FEL is quite adequate for the kiloWatt demonstrator, but not scalable even to the $100 \mathrm{~kW}$ device [3]. If we consider the high gain FEL amplifier, we find that there are no thermal loading limitations to increasing the average output power of this device up to the megawatt level. Since the amplification process develops in vacuum during one pass of the electron beam through the undulator, the problem of absorption of radiation in the cavity mirrors does not exist at all.

\section{FACILITY DESCRIPTION}

In this paper we perform design consideration of a MWscale UV FEL amplifier. Figure 1 shows the general scheme of the FEL amplifier. The arrangement of injector, which is practically identical to that designed at LBL for the CW-mode operation infrared FEL oscillator[6], is shown schematically in Fig. 2. The electron beam originates in a high-voltage DC gun with gridded thermionic cathode. The injector includes two subharmonic, roomtemperature buncher cavities and a $500 \mathrm{MHz}$ accelerating module. The bunched low-energy electron beam then enters the SRF linac for further acceleration up to energy of $1000 \mathrm{MeV}$. The accelerated beam enters the undulator, yields light, and finally decelerates through an energy recovery pass in the SRF linac before its remaining energy is absorbed in the beam dump at the final energy of about $10 \mathrm{MeV}$. In our conceptual design we assume the use of a conventional quantum laser as a master oscillator, which provides a continuous train of $10 \mathrm{ps}$ micropulses, with $100 \mathrm{~nJ}$ of optical energy per micropulse. The average output power of the master laser is about one Watt. A dye laser system with subsequent nonlinear optical elements pumped by $\mathrm{Nd}$ glass laser can be used for this purpose.

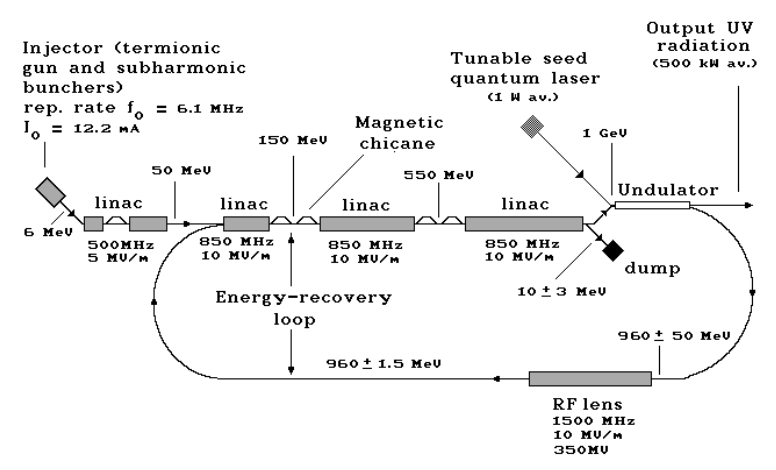

Figure 1: Schematic illustration of design configuration for the UV FEL amplifier 


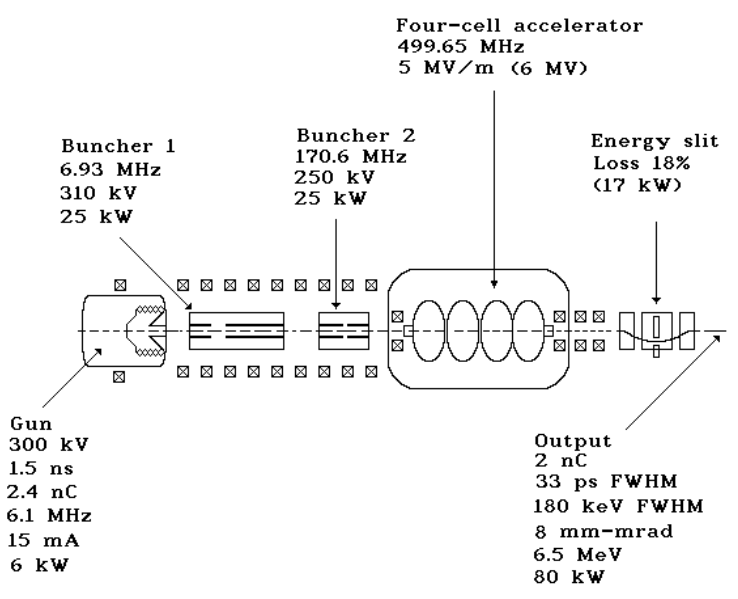

Figure 2: Schematic of the UV FEL amplifier injector

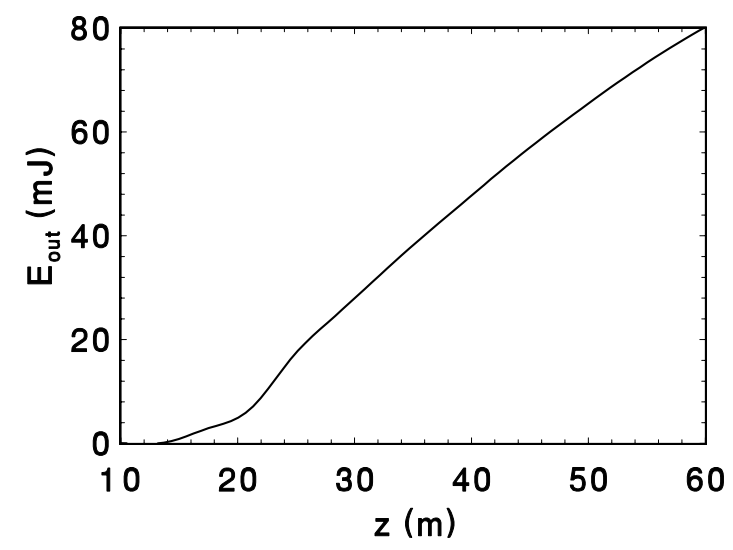

Figure 3: Energy in the radiation pulse versus the length for the FEL amplifier

In order to achieve the high peak current in the undulator the bunch must be compressed in a series of magnetic chicanes. The compression performs in three steps: at 30 $\mathrm{MeV}$ (from $4.2 \mathrm{~mm}$ to $1.6 \mathrm{~mm} \mathrm{rms}$ ), $150 \mathrm{MeV}$ (from 1.6 $\mathrm{mm}$ to $0.45 \mathrm{~mm} \mathrm{rms}$ ) and $550 \mathrm{MeV}$ (from $0.45 \mathrm{~mm}$ to 0.15 $\mathrm{mm} \mathrm{rms})^{1}$. Requirements for magnetic bunch compressors in our case are very close to those for magnetic bunch compressors in X-ray FELs [4]. In particular, the magnetic chicanes for the VUV FEL at TTF, which is presently under construction, is a good example for many problems related to our bunch compressor design [10].

Optimization of the parameters of the FEL amplifier has been performed with the three-dimensional time-dependent simulation code FAST [8]. This code has been developed in the framework of the investigations on X-ray FELs. The optimized parameters of UV FEL amplifier are presented in Table 1. A reliable method to increase the FEL efficiency

\footnotetext{
${ }^{1}$ The magnetic bunch compressors are arranged and located such that RF curvature, space charge effects, longitudinal wake fields do not limi the achievable bunch parameters
}

Table 1: Performance characteristics of the UV FEL amplifier

\begin{tabular}{|ll|}
\hline Electron beam & $1000 \mathrm{MeV}$ \\
Energy & $0.2 \%$ \\
rms energy spread & $2 \mathrm{nC}$ \\
Bunch charge & $0.5 \mathrm{ps}$ \\
rms pulse duration & $6.1 \mathrm{MHz}$ \\
Micropulse repetition rate & $8 \pi \mathrm{mm}-\mathrm{mrad}$ \\
normalized emittance & \\
Undulator & Planar \\
Type & $7.0 \mathrm{~cm}$ \\
Period & $12 \mathrm{~mm}$ \\
Gap & $1.15 \mathrm{~T}$ \\
Maximum peak field & 850 \\
\# of undulator periods & $3.0 \mathrm{~m}$ \\
External beta-function & \\
Radiation & $260-500 \mathrm{~nm}$ \\
Wavelength, & Transform-limited \\
Spectrum width & $110 \mathrm{GW}$ \\
Peak power & $0.5 \mathrm{MW}$ \\
Average power & $0.7 \mathrm{ps} \mathrm{(FWHM)}$ \\
Micropulse duration & $83 \mathrm{~mJ}$ \\
Micropulse energy & $6.1 \mathrm{MHz}$ \\
Repetition rate & \\
\hline
\end{tabular}

consists in an adiabatic change of the undulator parameter (or in undulator tapering). The length of untapered section of the undulator is equal to $18.4 \mathrm{~m}$. At the radiation wavelength $260 \mathrm{~nm}$, the peak value of magnetic field is about $1.15 \mathrm{~T}$. The field in the tapered section is reduced linearly with the length from $1.15 \mathrm{~T}$ down to the value of $1.05 \mathrm{~T}$ at the end of the undulator of $60 \mathrm{~m}$. Figure 3 presents evolution along the undulator length of the energy of the radiation pulse. Details regarding high-power UV FEL amplifier design can be found in [11].

The undulator for an industrial-use UV FEL amplifier needs to be rather long in order to provide the required power of the output radiation. Thus, strong focusing elements are needed for the electron beam transport system. A combined function undulator (CFU) approach [12], to implement strong focusing in a long undulator, is used in our design. Tolerances for field errors and alignment are estimated on the basis of work done for the $30 \mathrm{~m}$ long CFU undulator for the VUV FEL at the TESLA Test Facility [13].

The wall plug power to output optical power efficiency of a FEL for industrial applications is an important criterion. For the present design the ratio of the energy in the radiation pulse to the energy in the electron pulse is about $4 \%$. Energy recovery of most of the driver electron beam energy would increase the power efficiency. The energy of the spent electron beam is about $950 \mathrm{MeV}$. The energy spread of the electron beam after leaving the undulator is pretty large, about $\pm 50 \mathrm{MeV}$. An important feature of our design is that a very short electron bunch (of about 
$0.15 \mathrm{~mm} \mathrm{rms}$ ) is used for the generation of the radiation. When the electron bunch leaves the undulator, we apply a transformation of the particle distribution in the longitudinal phase space resulting in increased bunch length and decreased energy spread [7] ${ }^{2}$. After leaving the undulator the electron beam passes a debuncher (first arc). The length of the bunch is increased by a factor of about 30 , and the uncorrelated energy spread is transformed to one correlated with the position of the particles in the bunch. The resulting electron bunch length is still tolerable for use in the energy recovery scheme. Subsequently, the electron bunch passes a special $1500-\mathrm{MHz}$ SRF accelerating structure at $90^{\circ}$ crossing phase $\left(0^{\circ}\right.$ corresponding to running on-crest) and the energy spread of the electron beam is reduced to about $1.5 \mathrm{MeV}$. The electron bunch with such energy spread can be decelerated safely down to the energy of about $10 \mathrm{MeV}$, which is much less than the initial energy spread $^{3}$. As a result, we can reach the RF power to output optical power efficiency of about $80 \%$. Assuming the efficiency of the klystron modulator be $80 \%$, and the electronic efficiency of the high power CW klystron 60\%, we obtain that the $\mathrm{AC}$ wall plug power to output optical power efficiency is about $40 \%$. Another source of the energy consumption is cryogenic system of the SRF accelerator. The present design requires cooling of about 30 cryomodules. To do this, we need a He refrigerator with net power consumption about one MW. As a result, we obtain a total efficiency of the proposed UV FEL amplifier about to $20 \%$.

Driver linac cavities will use the energy recovery to reduce the RF power requirements to less than $5 \mathrm{~kW}$ per cavity. For our design we had decided to power cryomodule by one 23-kW klystron YK1180 from Siemens. Injector cavities and initial part of linac (first $50 \mathrm{MeV}$ accelerator section) operating without energy recovery, will require about $600 \mathrm{~kW}$ RF power. For this active part of the SRF linac we selected $500-\mathrm{MHz}$ SRF structure, since high power klystrons are commercially available for this frequency. An appropriate RF source is one $1000-\mathrm{kW}$ TH2105 tube.

\section{ACKNOWLEDGMENTS}

We thank J.R. Schneider, D. Trines and A. Wagner for their interest in this work, and B. Dwersteg, J. Pflüger, and J. Sekutowicz for many useful discussions and recommendations on technical topics.

\section{REFERENCES}

[1] G.R. Neil et al., Nucl. Instrum. and Methods A 358(1995) 159

[2] S.V. Benson et al., Nucl. Instrum. and Methods A 429(1999) 27

\footnotetext{
${ }^{2}$ The transformations are in the reverse order from those used for phase bunching

${ }^{3}$ In the present design the beam dump energy is below the photonneutron production threshold, so the problem of radio-nuclide production in the dump does not exist
}

[3] S.V. Benson et al., Nucl. Instrum. and Methods A 375(1996) ABS 4

[4] Conceptual Design of $500 \mathrm{GeV} \mathrm{e}^{+} \mathrm{e}^{-}$Linear Collider with Integrated X-ray Facility, DESY 1997-048

[5] The Linac Coherent Light Source (LCLS) Design Study Report, SLAC-R-521, 1998

[6] K.-J. Kim et al., LBL preprint Pub-5335(1992)

[7] A.J. Lichtenberg "Phase-Space Dynamics of Particles" John Willey and Sons, INC. New York-London-Sydney-Toronto 1969

[8] E.L. Saldin, E.A. Schneidmiller, and M.V. Yurkov, Nucl. Instrum. and Methods A 429(1999)233

[9] "A VUV Free Electron Laser at the TESLA Test Facility: Conceptual Design Report", DESY Print TESLA-FEL 9503, Hamburg, DESY, 1995

[10] T. Limberg et al., Nucl. Instrum. and Methods A 375(1996)322

[11] C. Pagani et al., "Design considerations of MW-Scale, High-Effisiency, Industrial-Use, Ultraviolet FEL Amplifier" DESY 00-015 (2000)

[12] J. Pflueger, Y.M. Nikitina Nucl. Instrum. and Methods A 381(1996)554

[13] J. Pflueger, H. Lu and T. Teichmann, Nucl. Instrum. and Methods A 429(1999)386 